\title{
MicroscopyPioneers
}

\section{Pioneers in Optics: Sir David Brewster (1781-1868)}

\section{Cameron Varano}

The Pennsylvania State University, 201 Old Main, University Park, PA 16802

cvarano@psu.edu

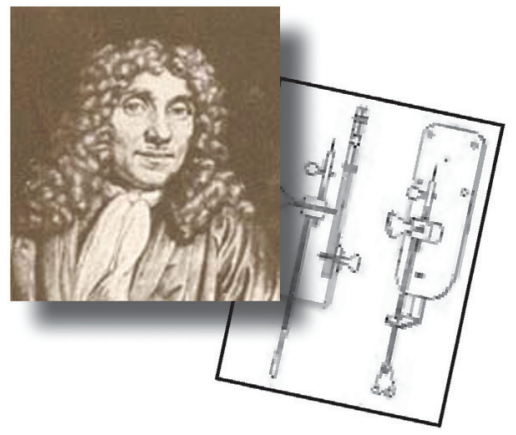

Note: This article is from the website Molecular Expressions ${ }^{T M}$, created by the late Michael Davidson and now maintained by Eric Clark, National Magnetic Field Laboratory, Florida State University, Tallahassee, FL 32306.

Sir David Brewster was a Scottish physicist who invented the kaleidoscope, made major improvements to the stereoscope, and discovered the polarization phenomenon of light reflected at specific angles. Brewster was born in Jedburgh, Scotland in 1781 and grew to become a brilliant student who entered the University of Edinburgh at the age of 12 to study the ministry. He was a prolific writer and became editor of the Edinburgh Magazine in 1802 and the Edinburgh Encyclopedia in 1808. In 1799, as a teenager, Brewster's interests turned to physics with a keen focus on optics, and he constructed several telescopes while dabbling in the physics of light. Brewster was a licensed minister of the Church of Scotland but never practiced this career, instead pursuing the finer aspects of optics and light.

Brewster's career blossomed while he was in his late twenties and early thirties. At that time, he was intensely pursuing details of the theory of light and wrote his first paper Some Properties of Light in 1813. One of Brewster's most important contributions to the science of physics was his work on polarization of light by reflection and biaxial crystals. To aid in his experiments, Brewster often constructed his own tools and even improved many technical instruments of the period.

In his studies on polarized light, Brewster discovered that when light strikes a reflective surface at a certain angle (now known as Brewster's Angle), the light reflected from that surface is plane-polarized. He elucidated a simple relationship between the incident angle of the light beam and the refractive index of the reflecting material. When the angle between the incident beam and the refracted beam equals 90 degrees, the reflected light becomes polarized. This rule is often used to determine the refractive index of materials that are opaque or available only in small quantities.

Brewster was elected to the Royal Society in 1815 and eventually was one of only a handful of scientists to be awarded all three principal medals of the Society. For his work in optics,
Brewster was awarded the Copley Medal in 1815, the Rumford Medal in 1818, and the Royal Medal in 1830 . He also was a founder of the British Association for the Advancement of Science.

An energetic enthusiast of color, Brewster invented the kaleidoscope in 1816 and patented it the following year. He published his extensive studies on the theory, design, and construction of kaleidoscopes in 1819 in a volume entitled Treatise on the Kaleidoscope. Apparently, there were some problems with the registration of his patent because he was not able to enforce infringements, and many companies began to offer custom versions of the kaleidoscope without paying royalties. The instrument ignited a craze in the early nineteenth century and quickly became a household toy for both children and adults alike.

Brewster was deeply interested in photography and had many conversations with Fox Talbot about the design of Talbot's Calotype process. He favored this process over the Daguerreotype and is quoted: "While a Daguerreotype picture is much more sharp and accurate in its details than a Calotype, the latter possesses the advantage of giving a greater breadth and massiveness to its landscapes and portraits."

Brewster wrote hundreds of papers on optics and designed a famous variation of the stereoscope, the Brewster Stereoscope. He studied the theory of this instrument and improved the performance by adding refractive lenses to his model. Brewster also wrote what many consider the definitive treatise on the stereoscope, The Stereoscope: Its History, Theory, and Construction. He also wrote his famous Treatise on Optics in 1831, and Memoirs of the Life, Writings, and Discoveries of Sir Isaac Newton in 1855.

He became Sir David Brewster in 1831 when he was knighted, and in the early 1840 s he was a major proponent in the use of Fresnel lenses in lighthouses. Brewster became principal of the United College of Saint Salvator and Saint Leonard of the University of Saint Andrews in 1838, and he became principal of the University of Edinburgh in 1859.

\section{Brewster's Angle and Polarized Light}

When considering the incidence of non-polarized light on a flat insulating surface, there is a unique angle at which the reflected light waves are all polarized into a single plane. This angle is commonly referred to as "Brewster's angle" and can 


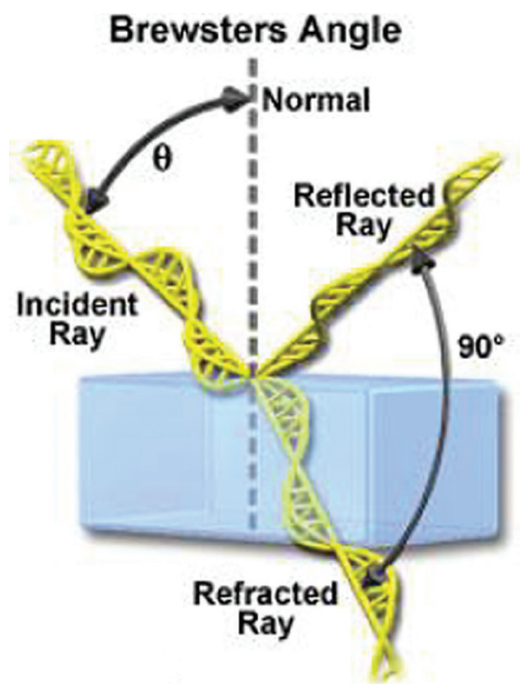

Figure 1: The principle of Brewster's angle.

be easily calculated using the following equation for a beam of light traveling through air:

$$
\mathbf{n}=\sin \left(\theta_{\mathrm{i}}\right) / \sin \left(\theta_{\mathrm{r}}\right)=\sin \left(\theta_{\mathrm{i}}\right) / \sin \left(\theta_{90-\mathrm{i}}\right)=\tan \left(\theta_{\mathrm{i}}\right)
$$

where $\mathbf{n}$ is the refractive index of the medium from which the light is reflected, $\boldsymbol{\theta}(\mathbf{i})$ is the angle of incidence, and $\boldsymbol{\theta}(\mathbf{r})$ is the angle of refraction. By examining the equation, it becomes obvious that the refractive index of an unknown specimen can be determined by the Brewster angle. This feature is particularly useful in the case of opaque materials that have high absorption coefficients for transmitted light, rendering the usual Snell's law formula inapplicable. Determining the amount of polarization through reflection techniques also eases the search for the polarizing axis of a sheet of polarizing film that is not marked.

The principle behind Brewster's angle is illustrated in Figure 1 for a single ray of light reflecting from the flat surface of a transparent medium having a higher refractive index than air. The incident ray is drawn with only two electric vector vibration planes but is intended to represent light having vibrations in all planes perpendicular to the direction of propagation. When the beam arrives on the surface at a critical angle (Brewster's angle; represented by the variable $\boldsymbol{\theta}$ in Figure 1), the polarization degree of the reflected beam is 100 percent, with the orientation of the electric vectors lying perpendicular to the plane of incidence and parallel to the reflected surface. The incidence plane is defined by the incident, refracted, and reflected waves. The refracted ray is oriented at a 90-degree angle from the reflected ray and is only partially polarized.

For water (refractive index of 1.333), glass (refractive index of 1.515), and diamond (refractive index of 2.417), the critical (Brewster) angles are 53, 57, and 67.5 degrees, respectively. Light reflected from a highway surface at the Brewster angle often produces annoying and distracting glare, which can be demonstrated quite easily by viewing the distant part of a highway or the surface of a swimming pool on a hot, sunny day. Modern lasers commonly take advantage of Brewster's angle to produce linearly polarized light from reflections at the mirrored surfaces positioned near the ends of the laser cavity.

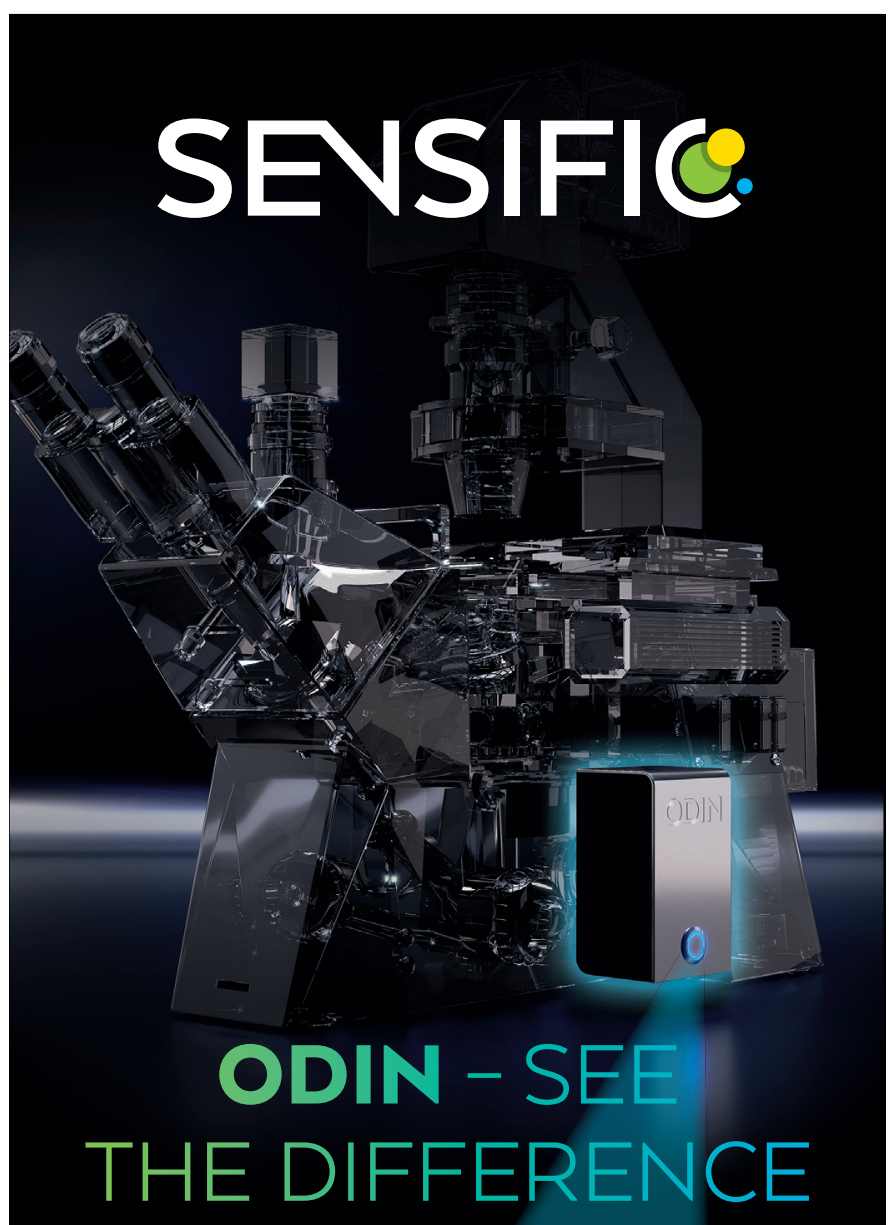

\section{ENHANCE YOUR MICROSCOPE}

$>$ high-speed . high-throughput

$>$ image . analyse . control . sort

$>$ droplets . cells . particles . algae . bacteria

$>$ bright-field . fluorescence , phase-contrast

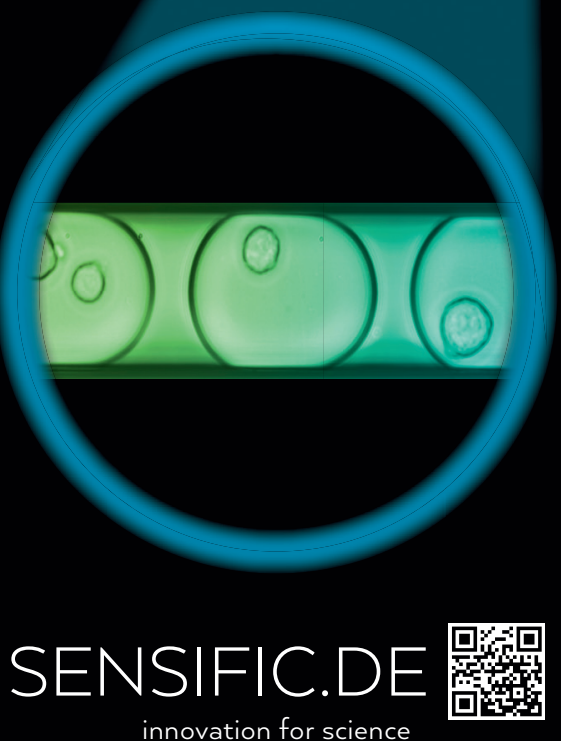

innovation for science 